Max-Planck-Institut für Bildungsforschung

Max Planck Institute for Human Development

This postprint was originally published by Elsevier as:

Wertz, A. E. (2019). How plants shape the mind. Trends in Cognitive Sciences, 23(7), 528-531. https://doi.org/10.1016/j.tics.2019.04.009

The following copyright notice is a publisher requirement:

(c) 2019. This manuscript version is made available under the CC-BY-NC-ND 4.0 license.

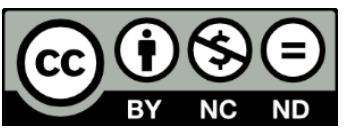

Provided by:

Max Planck Institute for Human Development - Library and Research Information

library@mpib-berlin.mpg.de 
This paper was published by Elsevier as:

Wertz, A. E. (2019). How plants shape the mind. Trends in Cognitive Sciences, 23(7), 528-531. https://doi.org/10.1016/j.tics.2019.04.009

\title{
How Plants Shape the Mind
}

\author{
Annie E. Wertz ${ }^{1}$,
}

Plants are easy to overlook in modern environments, but were a fundamental part of human life over evolutionary time. Recent work with infants suggests that the adaptive problems humans faced with respect to plants have left their mark on the human mind.

\section{Plants Are A(n Adaptive) Problem}

In many societies, plants are no longer a conspicuous part of human life. Plants are a part of the scenery outside and available for purchase, already packaged and processed, in grocery stores and garden centers. This limited contact with plants may seem perfectly normal, but across the entirety of human history it is quite unusual. Taking as a starting point the emergence of the genus Homo, humans spent $99 \%$ of our evolutionary history as hunter-gatherers. In a hunter-gatherer world, there were no such shops where the necessities of life could be easily acquired. Instead, our ancestors had to make a living by effectively utilizing the natural environment. Plants were an essential part of this process.

The archeological record and studies of modern hunter-gatherer and hunter-horticulturalist populations show that humans relied on plants in a variety of ways [1]. Plants are an important component of human diets, particularly the roots, fruits, and nuts of plants. Plant materials are used to construct a diverse array of artifacts and shelters. Plant chemicals are used to facilitate hunting and fishing, as well as in rituals and medicines. However, despite all of these benefits, plants can inflict serious costs. Plants have evolved an impressive set of defenses to protect against damage from herbivores [2]. All plants produce toxic chemical defenses, some of which can be harmful or even fatal to humans when ingested. Some plants also have mechanical defenses, such as thorns or stinging hairs, that can cause serious skin injury and in some cases systemic effects.

The problem is: how do humans figure out which plants are food (or otherwise useful) and which ones are fatal? This turns out to be a very difficult task. There are myriad plant species and herbivores that feed on them. The result of these complex coevolutionary relationships is that, from a human perspective, there are no morphological features common to all edible or toxic plants, even in the scale of the environments humans typically encounter without modern means of travel. Therefore, using general rules such as 'Avoid plants with white flowers' or 'Purple fruits are edible' simply would not work. In the former case, one would miss out on pears, and in the latter one would end up eating deadly nightshade. Importantly, the presence of difficult-to-detect and potentially fatal toxins makes learning about plants through trial and error sampling very costly. The best outcome for this process involves large amounts of wasted time and repeated exposure to noxious plant defenses. The worst-case scenario is death. These kinds of circumstances select for the evolution of social learning mechanisms [3].

The recurrent adaptive problems our species encountered over evolutionary time have shaped the human mind [4] and it is well known that plant defenses have structured the physiology and behavior of many 


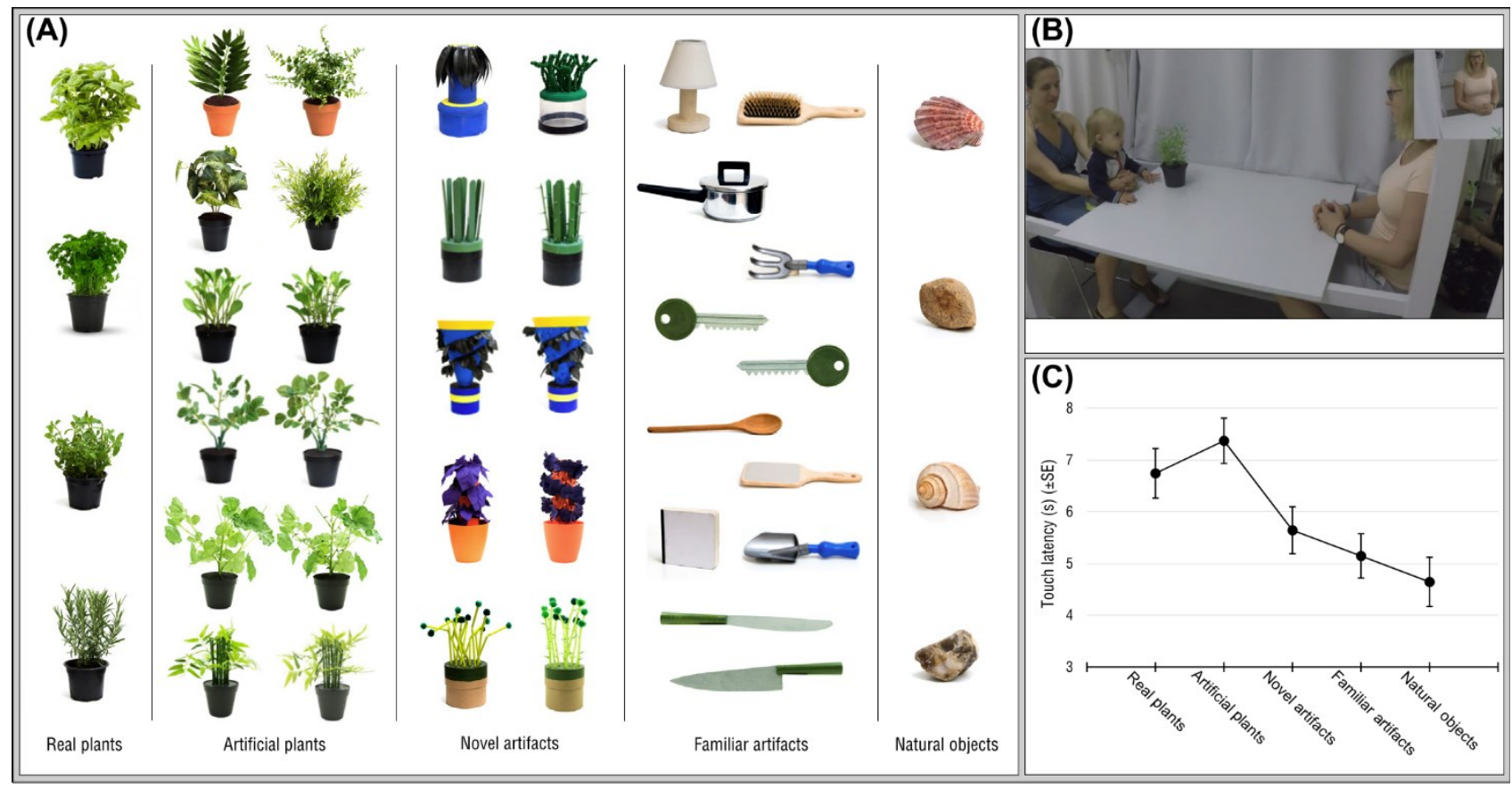

Trends In Cogntitheselemes

Figure 1. Testing Plant Learning and Avoidance of Natural Toxins (PLANT). (A) Some of the stimulus items used in our studies testing infants' behavioral avoidance of plants. The stimulus types include real plants, artificial plants that do not have a scent or change during the course of testing, novel artifacts matched to shape and/or color features of the plants, familiar artifacts some of which infants are typically allowed to handle themselves and others that are typically prohibited, and natural objects like shells and stones. (B) The experimental setup. The experimenter places each stimulus object in front of the infant in a counterbalanced order and maintains a neutral expression. Parents' eyes remain closed throughout the testing session to preclude their reactions to the stimuli influencing their child's behavior. Infants' reaching and looking behavior are later coded from videos of the session.

(C) Representative touch latency results from one of our studies. Adapted, with permission, from [11]. As predicted, infants take longer to reach out and touch plants (both real and artificial) than all of the other object types. We found a similar pattern of results in $[7,10]$.

animal species [2], including humans [5]. Accordingly, along with my colleagues, I have recently proposed a solution for the learning problems plants pose. We argue that the human mind contains a collection of behavioral avoidance strategies and social learning rules geared toward safely acquiring information about plants [6,7]. I will refer to this collection of cognitive systems as Plant Learning and Avoidance of Natural Toxins, or PLANT.

\section{Evidence for PLANT}

We have begun testing PLANT with studies of human infants. One line of work examines whether infants possess behavioral strategies that would mitigate plant dangers, similar to plant food rejections in older children [8]. Unlike the animate dangers that infants readily attend to (e.g., snakes, spiders [9]), dangerous plant toxins are difficult to detect but relatively easy to avoid. Plants are quite literally rooted to the spot and consequently can inflict harm only on individuals that come into contact with them. Therefore, we propose that PLANT includes behavioral avoidance strategies that protect infants by minimizing their physical contact with plants. To test this proposal, we present infants with plants and different kinds of control objects and measure their reaching behavior (Figure 1). Our results show that, as predicted, infants are reluctant to touch plants compared with other object types [7,10,11] and touch plants less frequently after making contact with them [10]. Infants are similarly avoidant of benign-looking plants and plants covered in sharp-looking thorns [10], suggesting that they initially treat all plants as potentially dangerous - a sensible strategy given that delicatelooking plants can be deadly poisonous.

Of course, not all plants can be avoided. Plant foods and materials must be foraged, which necessarily means coming into contact with plants. In some modern hunter-gatherer societies, plant foraging can begin as early as 2-3 years of age [1]. Therefore, in a second line of work, we are investigating whether infants are vigilant for social information about plants and use it to guide their behavior. These studies allow us to test the proposal that PLANT includes social learning rules. Thus far, we have found that infants look more often to adults when they first encounter plants, in the time before touching them [11], suggesting that behavioral 


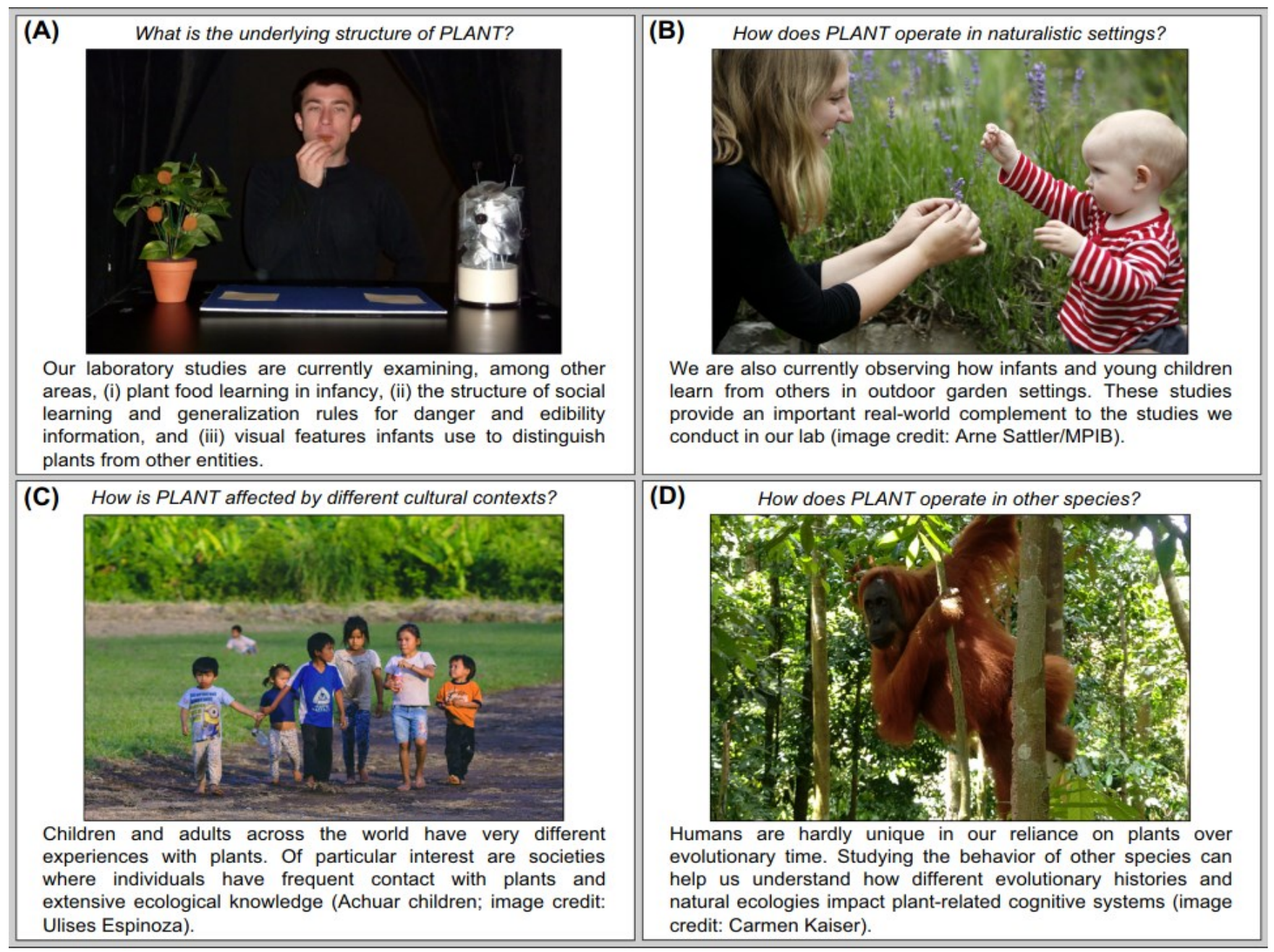

Figure 2. Open Questions and Future Directions for Plant Learning and Avoidance of Natural Toxins (PLANT). These lines of inquiry include (A) laboratory research, (B) naturalistic observations, (C) cross-cultural investigations, and (D) comparative studies.

avoidance strategies operate in concert with social learning processes. This structure would enable infants to observe signals from adults before making contact with potentially dangerous plants. Infants appear to be particularly attuned to social signals that allow them to learn which plants are edible [6,12]. In our studies, we show 6-and 18-montholds an adult eating pieces of fruit from a plant and a manmade object (Figure 2A). Despite seeing the same social information demonstrated with both object types, infants identify the plant, over the artifact, as a food source [6]. Once infants have learned that fruits from a particular plant are edible, 18-montholds generalize this information only to other plants that share the same leaf shape and fruit color [12]. This combination of social learning and restrictive generalization rules would prevent infants from inadvertently ingesting toxic plants.

\section{Seeing the Forest for the Trees}

Our empirical findings to date are consistent with the proposed PLANT systems. Infants appear to deploy a collection of behavioral avoidance strategies and social learning rules for plants. Consequently, PLANT minimizes infants' exposure to harmful plant defenses and allows them to safely acquire information about the specific plants they encounter from more knowledgeable individuals. In short, this work supports the claim that plants have shaped the human mind.

PLANT is a novel research area that provides fertile ground for future exploration (Figure 2). Of particular interest are cross- 
cultural studies that can shed light on the development of PLANT in different environments and comparative studies that can clarify the evolution of PLANT. Further, the integral role that plants played in human life and human evolution means that PLANT is likely to be enmeshed in a web of cognitive systems that support broader capacities like food learning, threat mitigation, categorization, and cultural transmission, among others. This interconnectedness makes PLANT an excellent starting point for future inquiry in these areas. At the same time, it is highly unlikely that infants, or adults for that matter, will treat plants as a special category in all circumstances. Research on cognitive systems like PLANT can provide new ways of exploring fundamental aspects of human cognition and understanding the evolution of learning.

\section{Acknowledgments}

This work was funded by the Max Planck Society.

${ }^{1}$ Max Planck Institute for Human Development, Max Planck

Research Group Naturalistic Social Cognition, Lentzeallee 94, 14195 Berlin, Germany

*Correspondence: wertz@mpib-berlin.mpg.de (A.E. Wertz). https://doi.org/10.1016/j.tics.2019.04.009

\section{References}

1. Hardy, K. and Martens, L.K., eds (2016) Wild Harvest: Plants in the Hominin and Pre-agrarian Human Worlds, Oxbow Books

2. Mithöfer, A. and Boland, W. (2012) Plant defense against herbivores: chemical aspects. Annu Rev. Plant Biol. 63, 431-450

3. Perreault, C. et al. (2012) A Bayesian approach to the evolution of social learning. Evol. Hum. Behav. 33, 449-459

4. Barrett, H.C. (2015) The Shape of Thought, Oxford University Press

5. Placek, C.D. and Hagen, E.H. (2015) Fetal protection: the roles of social learning and innate food aversions in South India. Hum. Nat. 26, 255-276

6. Wertz, A.E. and Wynn, K. (2014) Selective social learning of plant edibility in 6- and 18-month-old infants. Psychol. Sci. 24, 874-882

7. Wertz, A.E. and Wynn, K. (2014) Thyme to touch: infants possess strategies that protect them from dangers posed by plants. Cognition 130, 44-49

8. Lafraire, J. et al. (2016) Food rejections in children: cognitive and social/environmental factors involved in food neophobia and picky/fussy eating behavior. Appetite 96, 347-357

9. LoBue, V. et al. (2017) Developmental differences in infants' attention to social and nonsocial threats. Infancy 22 , $403-415$

10. Włodarczyk, A. et al. (2018) Every rose has its thorn: infants' behavioral responses to pointy shapes in naturalistic contexts. Evol. Hum. Behav. 39, 583-593

11. Elsner, C. and Wertz, A.E. (2019) The seeds of social learning: infants exhibit more social looking for plants than other object types. Cognition 183, 244-255

12. Wertz, A.E. and Wynn, K. (2019) Can I eat that too? 18month-olds generalize social information about edibility to similar looking plants. Appetite 138, 127-135 